Revista Brasileira de Agricultura Irrigada v.13, nº.3, p. 3444 - 3453, 2019

ISSN 1982-7679 (On-line)

Fortaleza, CE, INOVAGRI - http://www.inovagri.org.br

DOI: $10.7127 /$ rbai.v13n301057

Protocolo 1057.19 - 21/01/2019 Aprovado em 07/06/2019

\title{
POTENCIAL DE ÁGUA NO SOLO E FERTIRRIGAÇÃO NITROGENADA NA PRODUÇÃO E TROCAS GASOSAS DA ALFACE
}

Eduardo Augusto Agnellos Barbosa ${ }^{1}$, Erinelson Digner ${ }^{2}$, Wallyson Zarpellon Teixeira ${ }^{2}$, Rosana Fernandes Otto $^{3}$, Silvana Ohse ${ }^{3}$

\begin{abstract}
RESUMO
O estudo objetivou avaliar as trocas gasosas foliares, o crescimento da parte aérea e radicular de alface crespa, submetida a diferentes potenciais de água no solo $(-10,-20$ e $-30 \mathrm{kPa})$ e fertirrigação com nitrato de amônio e ureia. $\mathrm{O}$ experimento foi realizado em túnel alto no delineamento de blocos casualizado. Durante o ciclo de cultivo mensurou-se as trocas gasosas foliares com auxílio do analisador de gases por infravermelho portátil e estimou-se a eficiência instantânea do uso da água e eficiência de carboxilação. Na colheita determinou-se a altura, diâmetro da parte aérea, comprimento radicular e massa fresca da parte aérea. Aos 22 dias após o transplantio (DAT), a aplicação de nitrato de amônio elevou a assimilação líquida de $\mathrm{CO}_{2}$, a concentração de carbono intercelular e a eficiência instantânea de carboxilação em $16 \%, 7 \%$ e $24 \%$, respectivamente, em relação à ureia. Constatou-se que o potencial de $-21 \mathrm{kPa}$ elevou a assimilação líquida de $\mathrm{CO}_{2}$ em relação ao potencial de $-33 \mathrm{kPa}$ aos 22 DAT. Aos 36 DAT a fertirrigação com ureia no potencial de $-10 \mathrm{kPa}$ ocasionaou uma maior transpiração. $\mathrm{O}$ potencial de $-21 \mathrm{kPa}$ associado a fertirrigação com nitrato de amônio promoveu incremento da altura da alface e o potencial $-10 \mathrm{kPa}$ com fertirrigação de ureia elevou o diâmetro da parte aérea. As fontes de variação não ocasionaram efeitos significativos no comprimento radicular e massa fresca da parte aérea, os quais apresentaram valor médio de 13,89 cm e 211,14 $\mathrm{g} \mathrm{planta}^{-1}$, respectivamente.
\end{abstract}

Palavras-chave: Lactuca sativa L., capacidade de campo, fotossíntese, nitrato de amônio, ureia.

\section{WATER POTENTIALS OF SOIL AND NITROGEN FERTIRRIGATION IN THE PRODUCTION AND GAS EXCHANGES OF LETTUCE}

\footnotetext{
ABSTRACT

${ }^{1}$ Docente do Departamento de Ciência do Solo e Engenharia Agrícola da Universidade Estadual de Ponta Grossa, Campus Uvaranas, Ponta Grossa-PR. Email: eduardo.agnellos@gmail.com

${ }^{2}$ Acadêmico do curso de Agronomia da Universidade Estadual de Ponta Grossa, Campus Uvaranas, Ponta Grossa-PR. Email: erinelsondigner@hotmail.com; wally_zt@yahoo.com.br

${ }^{3}$ Docente do Departamento de Fitotecnia e Fitossanidade da Universidade Estadual de Ponta Grossa, Campus Uvaranas, Ponta Grossa-PR. Email: rfotto@uepg.br; sohse@uepg.br
} 
This study aims to evaluate leaf gas exchanges, shoot and root growth of lettuce, submitted to different soil water potentials $(-10,-20$ and $-30 \mathrm{kPa})$ and fertigation with ammonium nitrate and urea. The experiment was carried out in a high tunnel in the randomized block design. During the cultivation cycle, the foliar gas exchanges were measured using the portable infrared gas analyzer and estimated the instantaneous water use efficiency and carboxylation efficiency. At harvest, the height, shoot diameter, root length and shoot fresh weight were determined. At 22 days after transplanting (DAT), the ammonium nitrate application increased net $\mathrm{CO}_{2}$ assimilation, intercellular carbon concentration and instantaneous carboxylation efficiency in $16 \%, 7 \%$ and $24 \%$, respectively, in relation to urea. It observed that soil water potential of $-21 \mathrm{kPa}$ increased the net assimilation of $\mathrm{CO}_{2}$ in relation to the water potential of $-33 \mathrm{kPa}$ at $22 \mathrm{DAT}$. At $36 \mathrm{DAT}$, fertigation with urea in the matric potential of -10 $\mathrm{kPa}$ caused greater transpiration of plants. Fertigation with ammonium nitrate in the matric potential of $-21 \mathrm{kPa}$ increased the lettuce height and the $-10 \mathrm{kPa}$ potential with urea fertigation elevated shoot diameter. The sources of variation did not cause significant effects on root length and fresh shoot weight, which presented an average value of $13.89 \mathrm{~cm}$ and $211.14 \mathrm{~g} \mathrm{plant}^{-1}$, respectively.

Keywords: Lactuca sativa L., field capacity, photosynthesis, ammonium nitrate, urea.

\section{INTRODUÇÃO}

A alface (Lactuca sativa L.) é uma planta anual, proveniente da Ásia e pertencente à família da Asteraceae. É uma das hortaliças mais popular e consumida no Brasil, fonte de vitaminas, sais minerais e um produto de baixo valor calórico (OHSE et al., 2001).

Estudo com reposição hídrica na alface, como os realizados por Hamada e Testezlaf (1995), Valença et al. (2018) demonstram que o aumento do volume irrigado promove incrementos na produção. Por outro lado, Paulino et al. (2009) e Zhang et al. (2011) constataram que a irrigação excessiva ocasionou queda na qualidade da cultura.

O estabelecimento do limite superior de água no solo (LSAS) é fundamental para o adequado no manejo da irrigação e uso racional dos recursos hídricos e energia. Geralmente, adota-se a umidade na capacidade de campo como LSAS, a qual representa a água retida no solo após 2 ou 3 dias do solo ter sido saturado e praticamente ter cessado a drenagem livre (SSSA, 2008). Neste caso, considera-se apenas as características física do solo e não a cultura a ser irrigada. Ressalta-se que na prática é comum adotar a tensão de $10 \mathrm{kPa}$, como potencial matricial relacionado a umidade na capacidade de campo.

A irrigação por gotejamento mantém a umidade do solo próxima ao LSAS e apresenta elevada eficiência de aplicação e uniformidade, características básicas para o uso da fertirrigação (DUKES et al., 2010). A fertirrigação reduz as perdas de nutrientes em relação à adubação convencional, pois os fertilizantes são aplicados próximo ao sistema radicular e de forma ponderada durante o cultivo. Por outro lado, o manejo inadequado da irrigação e fertirrigação por gotejamento ocasiona perdas de nitrato por lixiviação e problemas de salinização do solo (MIN et al., 2016, TRIFONOV et al., 2018).

Em estudo com alface cv. Elba irrigada, Santos et al. (2018) relatam incremento linear na produtividade desta folhosa com o aumento da aplicação de $\mathrm{N}$ até a dose de $260 \mathrm{~kg} \mathrm{ha}^{-1}$. Desta forma, o manejo inadequado da irrigação em sistemas de produção da alface que requerem altas taxas de fertilizantes nitrogenados e possuem sistemas radiculares superficiais, podem resultar em perdas de $\mathrm{N}$ (CAHN; JOHNSON, 2017, ZHANG et al., 2019) e consequentemente apresentar quedas de rendimento desta cultura e contaminação dos aquíferos por nitrato. O estudo objetivou determinar o limite superior de água no solo da alface crespa fertirrigada com nitrato de amônio e ureia via gotejamento, por meio de parâmetros relacionados as trocas gasosas foliares, o crescimento da parte aérea e radicular e a massa fresca da parte aérea.

\section{MATERIAL E MÉTODOS}

O experimento foi conduzido em túnel alto, instalado na Fazenda Escola Capão da 
Onça da Universidade Estadual de Ponta Grossa (Fescon/UEPG), Ponta Grossa, PR (25 $05^{\prime} 31.3^{\prime \prime} \mathrm{S}, 50^{\circ} 03$ ' 28.0" O e altitude de 990 metros). Segundo a classificação climática de Köppen o clima da região é caracterizado como subtropical úmido, mesotérmico, do tipo $\mathrm{Cfb}$. O solo da área foi classificado como Cambissolo Háplico Típico (EMBRAPA, 2018).

A análise química do solo, realizada no Laboratório de Fertilidade do Solo da UEPG, apresentou as seguintes características: $\mathrm{pH}$ em $\mathrm{CaCl}_{2}$ igual a 5,0; $\mathrm{K}, \mathrm{Ca}$ e $\mathrm{Mg}$ trocável e CTC efetiva igual a $0,58,3,9,1,5$ e $6,54 \mathrm{cmolc} \mathrm{dm}^{-3}$, respectivamente; $\mathrm{P}$ igual a $22,9 \mathrm{mg} \mathrm{dm}^{-3}$, Corgânico igual a $25 \mathrm{~g} \mathrm{dm}^{-3}$ e saturação por base igual a $52 \%$. O delineamento experimental foi em blocos casualizados em esquema fatorial 3 x 2, com 3 blocos, totalizando dezoito unidades experimentais. A primeira fonte de variação foram três tensões de água no solo, que corresponderam aos potenciais matriciais de água no solo de $-10,-21$ e $-33 \mathrm{kPa}$. A segunda fonte de variação constituída por duas fontes distintas de nitrogênio, sendo nitrato de amônio e ureia, aplicados semanalmente via fertirrigação, seguindo a marcha de absorção da cultura (MARTINS et al., 2009). A dose de nitrogênio foram de $150 \mathrm{~kg} \mathrm{ha}{ }^{-1}$, conforme recomendação de Yuri et al. (2016). O transplantio das mudas de alface, cv. Crespa, foi realizado em 07 de novembro de 2016, no espaçamento de $0,30 \mathrm{~m}$ entre plantas e entre linhas de plantio. Cada parcela experimental foi composta por quatro linhas de plantio, com sete plantas em cada linha, totalizando 28 plantas de alface por parcela, consequentemente, cada parcela corresponde a uma área de $2,52 \mathrm{~m}^{2}$. O sistema de irrigação foi o gotejamento superficial, com emissores espaçados a $0,30 \mathrm{~m}$ e vazão de $1,5 \mathrm{~L} \mathrm{~h}^{-1}$. A injeção de fertilizantes na linha de irrigação foi realizada com auxilio de venture. No interior do túnel, foram construídos ramais de irrigação independentes, e assim, possibilitou a aplicação dos tratamentos individualizados em cada parcela experimental. Cada ramal foi composto por duas linhas de irrigação, instaladas entre a primeira e segunda linha de plantio e entre a terceira e quarta linha de plantio. Foram realizadas duas fertirrigações semanais. A reposição hídrica em cada tratamento foi realizada com auxílio do sensor HydroSense (Campbell Scientific) com haste de $12 \mathrm{~cm}$. Para estabelecer a relação entre a umidade volumétrica $(\theta \mathrm{i})$ e os potenciais matriciais $(\Psi \mathrm{m})$ definidos no estudo, instalaram-se quatro tensiômetros na camada de $0,0-10 \mathrm{~cm}$, após a formação dos canteiros, e estabeleceu-se previamente a relação entre $\Psi \mathrm{m}$ x $\theta \mathrm{i}$. Durante a condução do experimento, realizou-se novas aferições da relação $\Psi \mathrm{m} \times \theta \mathrm{i}$, devido ao rearranjo do solo. A determinação da umidade do solo foi realizada em dois pontos localizados na região central da parcela experimental. Posteriormente estabeleceu-se o tempo de irrigação para elevar a umidade do solo até os limites de potenciais estabelecidos no ensaio. As irrigações foram realizadas diariamente. Durante a condução do experimento realizouse duas avaliações de trocas gasosas, aos $22 \mathrm{e}$ 36 DAT. Utilizou-se o analisador de gases por infravermelho portátil (IRGA) modelo LI6400XT (Li-Cor), com área de câmara de 6,0 $\mathrm{cm}^{2}$, densidade de fótons fotossinteticamente ativos fixados em $1200 \mu \mathrm{mol} \mathrm{m} \mathrm{m}^{-2} \mathrm{~s}^{-1}$ e taxa de fluxo em $500 \mu \mathrm{mol} \mathrm{s}{ }^{-1}$, com concentração de $\mathrm{CO}_{2}$ na câmara em $370 \mathrm{ppm}$.

As avaliações foram realizadas no período das $9 \mathrm{~h} 30 \mathrm{~min}$ até $10 \mathrm{~h} 30 \mathrm{~min}$, em duas plantas por unidade experimental, localizadas na segunda e terceira linha de plantio e na região central da parcela. Os parâmetros analisados foram: a assimilação líquida de $\mathrm{CO}_{2}$ $(A)$ condutância estomática $(g s)$, transpiração foliar $(E)$ e concentração de carbono intercelular $(\mathrm{Ci})$. Por meio dos valores de trocas gasosas, estimou-se a eficiência instantânea do uso da água $(E i U A=\mathrm{A} / \mathrm{E})$ e a eficiência instantânea de carboxilação $(E i C=\mathrm{A} / \mathrm{Ci})$. A colheita da alface foi realizada no aos 40 DAT (17 de dezembro de 2016) e determinou-se, em quatro plantas por unidade experimental, a altura das plantas, o diâmetro da parte aérea, o comprimento do sistema radicular e a massa fresca da parte aérea. Os dados obtidos foram submetidos ao teste de Shapiro-Wilk. Após verificação da normalidade as variáveis respostas foram submetidas à análise de 
variância (ANOVA), e se $\mathrm{F}$ significativo ao nível de 5\% de significância, aplicou-se o teste de Tukey $(\mathrm{p}<0,05)$. As análises estatísticas foram realizadas no Software Sisvar (FERREIRA, 2014).

\section{RESULTADOS E DISCUSSÃO}

A análise de variância das trocas gasosas das folhas de alface aos 22 DAT encontra-se na
Tabela 1. Constatou-se que as fontes de nitrogênios aplicadas via fertirrigação promoveram efeitos significativos $(\mathrm{p}<0,05)$ na $A, C i$ e $E i C$.

Verificou também efeito significativo $\quad(p<0,05)$ dos $\quad$ potenciais água no solo sobre a A. Não foi observado efeito da interação nas variáveis relacionadas às trocas gasosas.

Tabela 1. Resumo da análise de variância e do teste de média para assimilação de $\mathrm{CO}_{2}\left(A, \mu\right.$ mol $\mathrm{CO}_{2}$ $\left.\mathrm{m}^{-2} \mathrm{~s}^{-1}\right)$, condutância estomática $\left(g s, \mathrm{~mol} \mathrm{~m} \mathrm{~s}^{-2} \mathrm{~s}^{-1}\right)$, transpiração foliar $\left(E, \mathrm{mmol} \mathrm{H}_{2} \mathrm{O} \mathrm{m}^{-2} \mathrm{~s}^{-1}\right)$, eficiência instantânea do uso da água (EiUA, mmol $\left.\mathrm{H}_{2} \mathrm{O} \mathrm{m}^{-2} \mathrm{~s}^{-1}\right)$ e eficiência instantânea de carboxilição (EiC, $\mu \mathrm{mol} \mathrm{m} \mathrm{s}^{-1}$ ), aos 22 dias após o transplantio (DAT) da alface, em túnel alto, Ponta Grossa-PR, 2017.

\begin{tabular}{ccccccccc}
\hline Fonte de variação & & $G L$ & $A$ & $g s$ & $E$ & $E i U A$ & $C i$ & $E i C$ \\
\hline \multirow{2}{*}{ Fonte N } & Ureia & & $10,00 \mathrm{~b}$ & 0,33 & 6,03 & 1,70 & $264,47 \mathrm{~b}$ & $0,0356 \mathrm{~b}$ \\
& NA & & $11,61 \mathrm{a}$ & 0,32 & 6,44 & 1,83 & $284,48 \mathrm{a}$ & $0,0451 \mathrm{a}$ \\
\hline Teste F $(\mathrm{N})$ & & 1 & $9,82^{*}$ & $0,14^{\mathrm{ns}}$ & $2,08^{\text {ns }}$ & $1,32^{\text {ns }}$ & $5,18^{*}$ & $8,77^{*}$ \\
\hline \multirow{2}{*}{$\Psi \mathrm{m}$} & $-10 \mathrm{kPa}$ & & $11,19 \mathrm{ab}$ & 0,33 & 6,21 & 1,81 & 275,45 & 0,041 \\
& $-21 \mathrm{kPa}$ & & $11,54 \mathrm{a}$ & 0,32 & 6,28 & 1,86 & 270,55 & 0,043 \\
& $-33 \mathrm{kPa}$ & & $9,70 \mathrm{~b}$ & 0,33 & 6,21 & 1,62 & 277,42 & 0,036 \\
\hline Teste F $(\Psi \mathrm{m})$ & & 2 & $4,83^{*}$ & $0,03^{\text {ns }}$ & $0,03^{\text {ns }}$ & $1,61^{\text {ns }}$ & $0,22^{\text {ns }}$ & $1,52^{\text {ns }}$ \\
\hline Teste F $\left(N^{*} \Psi \mathrm{m}\right)$ & & 2 & $0,21^{\text {ns }}$ & $1,14^{\text {ns }}$ & $0,03^{\text {ns }}$ & $0,40^{\text {ns }}$ & $0,12^{\text {ns }}$ & $0,12^{\text {ns }}$ \\
C.V. $(\%)$ & & & 14,22 & 24,69 & 13,64 & 19,94 & 9,61 & 9,61 \\
\hline
\end{tabular}

Valores seguidos por letras diferentes na coluna apresentam diferença significativa pelo teste de Tukey ( $<<0,05$ ). *Significativo a p $<0,05$; ${ }^{\text {ns }}$ Não significativo. $\mathrm{N}$, fontes de nitrogênio; NA, Nitrato de amônio; $\Psi \mathrm{m}$, potencial matricial de água no solo; C.V., coeficiente de variação; GL, Garu de liberdade.GL total $=35$.

A alface fertirrigada com nitrato de amônio, obteve $A$ de $11,61 \mu \mathrm{mol} \mathrm{CO}_{2} \mathrm{~m}^{-2} \mathrm{~s}^{-1}$, valor este superior em aproximadamente $16 \%$ ao observado nas plantas fertirrigadas com ureia, aos 22 DAT (Tabela 1). O nitrato de amônio apresenta as duas formas preferenciais de absorção de $\mathrm{N}$ pelas plantas e destaca-se que o íon $\mathrm{NH}_{4}{ }^{+}$é a forma prontamente disponível para assimilação pelas plantas (SOUZA; FERNANDES, 2006), já a ureia não é absorvida diretamente pelas plantas, precisando ser transformada em $\mathrm{NH}_{4}{ }^{+}$e posteriormente em $\mathrm{NO}_{3}{ }^{-}$(ROCHETTE et al., 2009). Ressalta-se, que os valores médios de $A$ aos 22 DAT, observado neste estudo, encontram-se próximos aos valores observados aos 28 DAT por Fátima et al. (2018) na cultura da Alface, cv. Crespa, cultivada em casa de vegetação e com reposição de $100 \%$ da evapotranspiração real. Durante as medições de trocas gasosas foliares aos $22 \mathrm{DAT}$, contatou-se maior $C i$ nas plantas fertirrigadas com nitrato de amônio
(Tabela 1), este resultado sugere que momentos antes da leitura as alface submetida a aplicação deste fertilizante apresentavam maior assimilação de $\mathrm{CO}_{2}$, fato constatado pelos valores médios de $A$. Mesmo com maior concentração de carbono no mesófilo foliar, a alface crespa ferirrigada com nitrato de amônio apresentou eficiência de carboxilação $24 \%$ superior as plantas fertirrigadas com uraia, indicando baixa limitação à fotossíntese (TAIZ; ZEIGER, 2013) das plantas fertirrigadas com o nitrato de amônio.

O aumento no valor de $\mathrm{Ci}$, comumente é associado a menor restrição estomática das plantas (TOMINAGA et al., 2018) favorecendo a $A$, porém, não foi constatado efeitos significativos para $g s$. Como o valor de $C i$ indica essencialmente o substrato de $\mathrm{CO}_{2}$ disponível para o processo fotossintético (FARQUHAR et al., 1980), constata-se, uma maior eficiência do aparelho fotossintético da alface submetida a aplicação de nitrato de 
amônio até os 22 DAT. Este fato é reforçado pela maior $E i C$ das plantas fertirrigadas com nitrato de amônio em relação à aplicação de ureia (Tabela 1).

Por meio dos valores de EiC, constata-se uma maior taxa de carboxilação e sugere maior quantidade ou atividade da Rubisco (WULLSCHLEGER, 1993). A análise de variância para as trocas gasosas aos 36 DAT encontra-se na Tabela 2. Por meio da análise de desdobramento da interação para a transpiração foliar, aos 36 DAT (Tabela 3), observa-se efeito significativo dos potenciais de água no solo para a fertirrigação com ureia. Já a alface fertirrigada com nitrato de amônio não apresentou diferenças significativas na $\mathrm{E}$ para os diferentes potencias de água no solo. Para as fontes de $\mathrm{N}$, constatou-se efeito significativo $(\mathrm{p}<0,05)$ no potencial matricial de $-10 \mathrm{kPa}$.

Tabela 2. Resumo da análise de variância e do teste de média para assimilação de $\mathrm{CO}_{2}\left(A, \mu\right.$ mol m${ }^{-2}$ $\left.\mathrm{s}^{-1}\right)$, condutância estomática $\left(g s, \mathrm{~mol} \mathrm{~m} \mathrm{~m}^{-2}\right)$, transpiração foliar $\left(\mathrm{Tr}, \mathrm{mmol} \mathrm{H} \mathrm{O}_{2} \mathrm{O} \mathrm{m}^{-2} \mathrm{~s}^{-1}\right)$, eficiência do uso da água (EUA, mmol $\mathrm{H}_{2} \mathrm{O} \mathrm{m}^{-2} \mathrm{~s}^{-1}$ ) e eficiência instantânea de carboxilação ( $\mathrm{E} / \mathrm{Ci}, \mu \mathrm{mol} \mathrm{m} \mathrm{m}^{-1}$ ), aos 36 dias após o transplantio (DAT) da alface, em túnel alto, Ponta Grossa-PR, 2017.

\begin{tabular}{|c|c|c|c|c|c|c|c|c|}
\hline & & GL & $A$ & $g s$ & $E$ & $E i U A$ & $\mathrm{Ci}$ & $E i C$ \\
\hline \multirow{2}{*}{ Fonte N } & Ureia & & 9,56 & 0,32 & 4,78 & 2,04 & 245,14 & 0,0382 \\
\hline & NA & & 8,86 & 0,33 & 4,85 & 1,84 & 248,55 & 0,0360 \\
\hline Teste F (N) & & 1 & $0,89^{\mathrm{ns}}$ & $0,02^{\mathrm{ns}}$ & $0,13^{\mathrm{ns}}$ & $3,10^{\mathrm{ns}}$ & 1,09 & $0,85^{\mathrm{ns}}$ \\
\hline \multirow{3}{*}{$\Psi \mathrm{m}$} & $-10 \mathrm{kPa}$ & & 9,49 & 0,32 & $4,96 \mathrm{ab}$ & 1,89 & 246,57 & 0,0375 \\
\hline & $-21 \mathrm{kPa}$ & & 9,30 & 0,33 & $5,04 \mathrm{a}$ & 1,84 & 247,08 & 0,0371 \\
\hline & $-33 \mathrm{kPa}$ & & 9,21 & 0,33 & $4,51 \mathrm{~b}$ & 2,07 & 247,17 & 0,0367 \\
\hline Teste F ( $\Psi \mathrm{m})$ & & 2 & $0,09^{\text {ns }}$ & $0,17^{\mathrm{ns}}$ & $3,76^{*}$ & $1,41^{\mathrm{ns}}$ & $0,03^{\mathrm{ns}}$ & $0,04^{\mathrm{ns}}$ \\
\hline Teste F $(\mathrm{N} * \Psi \mathrm{m})$ & & 2 & $2,15^{\mathrm{ns}}$ & $0,22^{\mathrm{ns}}$ & $3,98 *$ & $0,67^{\mathrm{ns}}$ & $1,61^{\mathrm{ns}}$ & $2,20^{\mathrm{ns}}$ \\
\hline C.V. $(\%)$ & & & 9,86 & 18,04 & 11,84 & 17,83 & 2,81 & 14,98 \\
\hline
\end{tabular}

Valores seguidos por letras diferentes na coluna apresentam diferença significativa pelo teste de Tukey ( $<<0,05$ ). *Significativo a p $<0,05$; ${ }^{\text {ns }}$ Não significativo. $\mathrm{N}$, fontes de nitrogênio; NA, Nitrato de amônio; $\Psi \mathrm{m}$, potencial matricial de água no solo; C.V., coeficiente de variação. ; GL, Garu de liberdade; GL total = 35 .

Tabela 3. Análise de desdobramento da interação para a transpiração foliar (E, mmol $\left.\mathrm{H}_{2} \mathrm{O} \mathrm{m}^{-2} \mathrm{~s}^{-1}\right)$, aos 36 dias após o transplantio (DAT) da alface, em túnel alto, Ponta Grossa-PR, 2017.

\begin{tabular}{ccccc}
\hline F.V. & \multicolumn{3}{c}{$\Psi \mathrm{m}$} & \multirow{2}{*}{ Teste F } \\
\hline Fonte N & $-10 \mathrm{kPa}$ & $-21 \mathrm{kPa}$ & $-33 \mathrm{kPa}$ & \\
\hline Ureia & $5,32 \mathrm{Aa}$ & $4,88 \mathrm{Aab}$ & $4,17 \mathrm{Ab}$ & \\
NA & $4,62 \mathrm{Ba}$ & $5,15 \mathrm{Aa}$ & $4,74 \mathrm{Aa}$ & $1,56^{\mathrm{ns}}$ \\
\hline Teste F & $4,75^{*}$ & $0,65^{\mathrm{ns}}$ & $2,96^{\mathrm{ns}}$ &
\end{tabular}

Valores seguidos por letras minúsculas na linha e maiúscula na coluna apresentam diferenças significativas pelo teste de

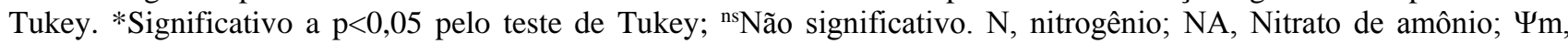
potencial matricial de água no solo.

No manejo da fertirrigação com ureia, a redução dos potenciais de água no solo, aos 36 DAT, promoveu um declínio na transpiração foliar da alface (Tabela 3). As plantas submetidas ao potencial de $-10 \mathrm{kPa}$ apresentaram um $\mathrm{E}$ de 5,32, sendo superior a $E$ média das plantas submetidas ao tratamento de $-33 \mathrm{kPa}(4,17)$. Os valores de $E$ estão próximos aos observados por Santos et al. (2010) na cultura da alface, aos 41 DAT.

Para o manejo da irrigação no $\Psi \mathrm{m}$ de -10 $\mathrm{kPa}$, constata-se por meio da análise de desdobramento que a plantas fertirrigadas com ureia tiveram um incremento de $15 \%$ na $E$, em relação a aplicação de nitrato de amônio, aos 36 DAT (Tabela 3). 
Destaca-se que plantas com maior taxa transpiratória tende a ter uma maior absorção de nutriente via fluxo em massa (TANGUILIG et al., 1987).

A análise de variância das respostas morfológicas da alface na colheita está ilustrada na Tabela 4. Constataram-se efeitos significativos da interação $(\mathrm{p}<0,05)$ para a altura e diâmetro da parte aérea. Não foram constatados efeitos isolados das fontes de variação sobre as variáveis biométricas no momento da colheita da alface.

Tabela 4. Resumo da análise de variância e do teste de média para a altura $(\mathrm{cm})$, diâmetro da parte aérea (DPA, cm), comprimento radicular $(\mathrm{CR}, \mathrm{cm})$ e massa fresca da parte aérea (MFPA, g planta $\left.{ }^{-1}\right)$ na colheita da alface cultivada em túnel alto, Ponta Grossa-PR.

\begin{tabular}{ccccccc}
\hline Tratamento & & GL & Altura & DPA & CR & MFPA \\
\hline Fonte N & Ureia & & 22,69 & 36,92 & 13,75 & 209,39 \\
& NA & & 23,20 & 35,97 & 14,03 & 212,89 \\
\hline Teste F $(\mathrm{N})$ & & 1 & $0,57^{\text {ns }}$ & $0,65^{\text {ns }}$ & $0,20^{\text {ns }}$ & $0,08^{\text {ns }}$ \\
\hline & $-10 \mathrm{kPa}$ & & 23,04 & 35,79 & 13,54 & 209,75 \\
& $-21 \mathrm{kPa}$ & & 22,91 & 36,79 & 14,36 & 208,58 \\
& $-33 \mathrm{kPa}$ & & 22,88 & 36,75 & 13,80 & 215,08 \\
\hline Teste F $(\Psi \mathrm{m})$ & & 2 & $0,02^{\text {ns }}$ & $0,31^{\text {ns }}$ & $0,55^{\text {ns }}$ & $0,10^{\text {ns }}$ \\
\hline Teste F $\left(\mathrm{N}^{*} \Psi \mathrm{m}\right)$ & & 2 & $4,42^{*}$ & $3,20^{*}$ & $2,08^{\text {ns }}$ & $2,37^{\text {ns }}$ \\
C.V. $(\%)$ & & & 12,20 & 16,63 & 17,46 & 18,78 \\
\hline
\end{tabular}

*Significativo a p $<0,05 ;{ }^{\text {ns }}$ Não significativo. N, fontes de nitrogênio; NA, Nitrato de amônio; P, potencial de água no solo; C.V., coeficiente de variação. GL, Garu de liberdade; GL total $=35$.

Por meio da análise de desdobramentos, observou-se efeito das fontes de $\mathrm{N}$ sobre a altura das plantas apenas no potencial de -21 $\mathrm{kPa}$ (Tabela 5). Verifica-se que a aplicação de nitrato de amônio no potencial de $-21 \mathrm{kPa}$ elevou a altura da alface em aproximadamente
$12 \%$, em relação à fertirrigação com ureia. De modo geral, a altura das plantas de alface encontram-se próximas aos valores observados por Magalhães et al. (2015) ao irrigar as cultivares Rapids e Simpson (tipo crespas) com $100 \%$ da evapotranspiração da cultura.

Tabela 5. Análise de desdobramento da interação fonte de $\mathrm{N} x$ tensão de água no solo para a altura da planta $(\mathrm{cm})$ e o diâmetro da parte aérea (DPA, cm), aos 36 dias após o transplantio (DAT) da alface, em túnel alto, Ponta Grossa-PR, 2017.

\begin{tabular}{|c|c|c|c|c|}
\hline F.V. & \multicolumn{3}{|c|}{$\Psi \mathrm{m}$} & \multirow{2}{*}{ Teste F } \\
\hline Fonte N & $-10 \mathrm{kPa}$ & $-21 \mathrm{kPa}$ & $-33 \mathrm{kPa}$ & \\
\hline \multicolumn{5}{|c|}{ Altura } \\
\hline Ureia & $24,08 \mathrm{Aa}$ & $21,58 \mathrm{Ba}$ & $22,41 \mathrm{Aa}$ & $1,96^{\mathrm{ns}}$ \\
\hline NA & $22,00 \mathrm{Aa}$ & $24,25 \mathrm{Aa}$ & $23,33 \mathrm{Aa}$ & $2,48^{\mathrm{ns}}$ \\
\hline Teste F & $3,23^{\mathrm{ns}}$ & $5,44 *$ & $0,64^{\mathrm{ns}}$ & \\
\hline \multicolumn{5}{|c|}{ DPA } \\
\hline Ureia & $38,33 \mathrm{Aa}$ & $36,50 \mathrm{Aa}$ & $35,91 \mathrm{Aa}$ & $1,77^{\mathrm{ns}}$ \\
\hline NA & $33,25 \mathrm{Ba}$ & $37,08 \mathrm{Aa}$ & $37,58 \mathrm{Aa}$ & $2,73^{\mathrm{ns}}$ \\
\hline Teste F & $6,28 *$ & $0,08^{\mathrm{ns}}$ & $0,68^{\text {ns }}$ & \\
\hline
\end{tabular}

Valores seguidos por diferentes letras minúsculas na linha e maiúscula na coluna apresentam diferenças significativas pelo teste de Tukey $(\mathrm{p}<0,05)$. *Significativo a $\mathrm{p}<0,05 ;{ }^{\text {ns }}$ Não significativo. N, nitrogênio; NA, Nitrato de amônio; $\Psi \mathrm{m}$, potencial matricial de água no solo.

Com o desdobramento da análise de interação, constatou-se efeito significativo sobre o DPA da alface $(\mathrm{p}<0,05)$ para as fontes de $\mathrm{N}$ no potencial de $-10 \mathrm{kPa}$, com a fertirrigação com ureia se destacando em relação às plantas fertirrigadas com nitrato de 
amônio. Salienta-se que as plantas fertirrigadas com ureia no potencial matricial de $-10 \mathrm{kPa}$ apresentaram também maior transpiração foliar aos 36 DAT (Tabela 3). Incrementos na transpiração favorece a absorção de nutrientes (TANGUILIG et al., 1987) e, consequentemente, o desenvolvimento da parte aérea. Entretanto, o maior DPA não resultou em efeitos significativos na massa fresca da parte aérea das plantas (Tabela 4).

Por meio dos resultados da MFPA, constata-se a ausência de efeitos significativos $(\mathrm{p}<0,05)$ dos potenciais de água no solo e dos fertilizantes nitrogenados aplicados via fertirrigação (Tabela 4). Galbiatti et al. (2007), estudando por três ciclos os efeitos de diferentes fertilizantes e qualidades de água de irrigação na alface crespa, não observaram efeitos na massa MFPA das plantas. Carvalho (2013) em estudo com a cultura da alface, em ambiente protegido, aplicando diferentes tensões de água no solo $(-15$ a $-60 \mathrm{kPa})$ e diferentes doses de ureia, não observou efeitos significativos na produção de alface.

O comprimento radicular não apresentou diferenças significativas para os potenciais de água no solo e fontes de N (Tabela 4). Pinto et al. (2017) avaliando o CR da alface crespa irrigada por microaspersão, obtiveram valor médio de CR próximo a $13,3 \mathrm{~cm}$. Sutton e Merit (1993) observaram que a densidade de raiz da alface irrigada por gotejamento e com manutenção da umidade próxima a capacidade de campo, concentra-se na camada de $0-15 \mathrm{~cm}$. Em estudo com avaliação da profundidade do sistema radicular da alface irrigada por diferentes sistemas de irrigação, Jackson e Stivers (1993) observaram variações na profundidade efetiva do sistema radicular. $\mathrm{Na}$ irrigação por sulco e aspersão, os autores verificaram maior concentração de raízes da alface na camada de $0-50 \mathrm{~cm}$. Nas áreas irrigadas por gotejamento superficial e subsuperficial as raízes se concentraram próxima ao gotejador e na profundidade de 15 $\mathrm{cm}$.

Destaca-se que o manejo da irrigação foi realizado com sensor de haste de $12 \mathrm{~cm}$.

\section{CONCLUSÕES}

O cultivo da alface crespa fertirrigada via gotejamento superficial com nitrato de amônio em condições de túnel alto elevou a fotossíntese líquida desta folhosa aos 22 DAT.

A aplicação deste fertilizante considerando o limite superior de água no solo no potencial mátrico de $-21 \mathrm{kPa}$ promoveu uma maior altura das plantas. A alface crespa fertirrigada no potencial matricial $-10 \mathrm{kPa}$ associado a fertirrigação com ureia promoveu uma maior transpiração foliar aos 36 dias após o transplantio e elevou o diâmetro da parte aérea da alface.

O manejo da irrigação considerando os potenciais mátricos de $-10,-20$ e $-30 \mathrm{kPa}$ como limites superiores de água no solo e a fertirrigação com nitrato de amônio e ureia no cultivo da alface crespa não ocasionaram alterações na massa fresca da parte aérea e no comprimento radicular

\section{AGRADECIMENTOS}

A Fundação Araucária pela concessão da bolsa de inciação científica ao segundo autor e a Fazenda Escola Capão da Onça da UEPG pelo auxílio no desenvolvimento do estudo.

\section{REFERÊNCIAS BIBLIOGRÁFICAS}

CAHN, M.; JOHNSON, L.F. New approaches to irrigation scheduling of vegetables. Horticulturae, v.3, n.28, 1-20, 2017. https://doi.org/10.3390/horticulturae3020028

\section{CARVALHO, K. S. Alface Americana submetida à adubação nitrogenada e tensões de água no solo em ambiente protegido. 2013. 54f. Dissertação (Mestrado em Engenharia Agrícola) - Universidade Federal do Mato Grosso, Rondonópolis.}

DUKES, M. D.; ZOTARELLI, L.; MORGAN, K. T. Use of irrigation for vegetable crops in Florida. HortTechnology, v.20, n.1, p.133142 , 2010. https://doi.org/10.21273/HORTTECH.20.1.133 
EMBRAPA, Empresa Brasileira de Pesquisa Agropecuária. Centro nacional de Pesquisa de Solos. Sistema brasileiro de classificação de solos. 5 ed. Brasília:Embrapa, 2018. 356 p.

FÁTIMA, R. T.; JESUS, E. G.; GUERRERO, A. C.; ROCHA, J. L. A.; BRITO, M. E. B. Crescimento e trocas gasosas em alface cultivada sob regimes hídricos e adubação fosfatada. Revista Brasileira de Agricultura Irrigada. v.12, n.3, p.2683-2691, 2018. https://doi.org/10.7127/rbai.v12n300854

FARQUHAR, G.D.; VON CAEMMERER, S.; BERRY, J. A. A biochemical model of photosynthetic $\mathrm{CO}_{2}$ assimilation in leaves of C3 species. Planta, v.149, p.78-90, 1980. https://doi.org/10.1007/BF00386231

FERREIRA, D. F. Sisvar: a Guide for its Bootstrap procedures in multiple comparisons. Ciência e Agrotecnologia, v.38, n.2, p.109112, 2014. https://doi.org/10.1590/S141370542014000200001

GALBIATTI, J. A.; CAVALCANTE, I, H, L.; RIBEIRO, A. G.; BECKMANN, M. Z. Fertilização e qualidade da água de irrigação no crescimento e desenvolvimento da alface. Scientia Agraria, v.8, n.2, p.185-192, 2007. https://doi.org/10.5380/rsa.v8i2.8368

HAMADA, E; TESTEZLAF, R. Desenvolvimento e produtividade da alface submetida a diferentes lâminas de água através da irrigação por gotejamento. Pesquisa Agropecuária. Brasileira, v.30 n.9, p.12011209, 1995.

JACKSON, L.E.; STIVERS, L.J. Root distribution of lettuce under commercial production: implications for crop uptake of nitrogen. Biological Agriculture \& Horticulture, v.9, n.3, 273-293, 1993. https://doi.org/10.1080/01448765.1993.97546 39.

MAGALHÃES, F. F.; CUNHA, F. F.; GODOY, A. R.; SOUZA, E. J.; SILVA, T. R. Produção de cultivares de alface tipo crespa sob diferentes lâminas de irrigação. WRIM, v.41, p.41-50, 2015. https://doi.org/10.19149/23166886/wrim.v4n1-3p41-50

MARTINS, C. M.; MEDEIROS, J. F.; LOPES, W. A. R.; BRAGA, D. F.; AMORIM, L. B. Curva de absorção de nutrientes em alface hidropônica. Revista Caatinga, v.22, n.4, p.123-128, 2009.

MIN, W.; GUO, H.; ZHOU, G.; ZHANG, W.; MA, L.; YE, J.; HOU, Z.; WU, L. Soil salinity, leaching, and cotton growth as affected by saline water drip irrigation and $\mathrm{N}$ fertigation, Acta Agriculturae Scandinavica, Section B — Soil \& Plant Science, v.66, n.6, p.489-501, 2016. https://doi.org/10.1080/09064710.2016.11811 99

OHSE, S.; DOURADO-NETO, D.; MANFRON, P. A.; SANTOS, O.S. Qualidade de cultivares de alface produzidos em hidroponia. Scientia Agricola, v.58, n.1, p.181-185, 2001. https://doi.org/10.1590/S010390162001000100027

PAULINO, M. A. O.; FIGUEIREDO, F. P.; VIEIRA, E. O.; FERNANDES, R. C.; MAIA, J. T. L. S.; GUILHERME, D. O. Manejo da água no cultivo de alface irrigado pelo sistema de microaspersão. Revista Brasileira de Agricultura Irrigada, v.3, n.1, p.22-29, 2009. https://doi.org/10.7127/rbai.v3n100010.

PINTO, A. A.; SANTANA, L .D.; CAMARA, F. T.; RODRIGUES, W. A. D.; COSTA, M. N .F.; PENHA FILHO, N. Desenvolvimento e produção de alface crespa utilizando culturas para sombreamento lateral. Revista Verde de Agroecologia e Desenvolvimento Sustentável. v.12, n.4, p.665-660, 2017. https://www.gvaa.com.br/revista/index.php/R VADS/article/view/4840/4616.

ROCHETTE, P.; MacDONALD, J. D.; ANGERS, D.; CHANTINI, M. H.; GASSER, M.; BERTRAND, N. Banding urea increased ammonia volatilization in a dry acidic soil. 
Journal Environmental Quality, v.38, p.1383-1390, 2009. https://doi.org/10.2134/jeq2008.0295.

SANTOS, C. M.; GONÇALVES, L. E.; GOMES, T. C. A.; JADOSKI, C. J.; NASCIMENTO, L. A.; SANTOS, E. D. Photosynthetic measurements in lettuce submitted to different agroindustrial residue composting. Pesquisa Aplicada \& Agrotecnologia, v.3, p. 103-112, 2010. https://doi.org/10.5777/paet.v3i3.1269

SANTOS, J. R. C.; FERNANDES, C. N. V.; OLIVEIRA FILHO, J. N.; SILVA, A. R. A.; FERNANDES, J. N. V.; SARAIVA, K. R. Adubação nitrogenada e cobertura do solo no cultivo da alface irrigada. Revista Brasileira de Agricultura Irrigada, v. 12, n.1, p.23272337 , 2018. https://doi.org/10.7127/rbai.v12n100702

SSSA - Soil Science Society of America. Glossary of soil science terms 2008. SSSA:Madison, 2008. 88p.

SOUZA, S. R.; FERNANDES, M. S. Nitrogênio. In: FERNANDES, M. S. Nutrição Mineral de Plantas. Viçosa:Editora Sociedade Brasileira de Ciência do Solo, 2006. Cap. 9. p. 216-252.

SUTTON, B. G.; MERIT, N. Maintenance of lettuce root zone at field capacity gives best yields with drip irrigation. Scientia Horticulturae, v.56, n.1, p.1-11, 1993.

TAIZ, L.; ZEIGER, E. Fisiologia vegetal. $5^{\text {a }}$ ed. Porto Alegre: Artmed, 918p. 2013.

TANGUILIG, V. C.; YAMBAO, E. B.; O'TOOLE, J. C.; DE DATTA, S. K. Water stress effects on leaf elongation, leaf water potential, transpiration, and nutrient uptake of rice, maize, and soybean. Plant Soil, v.103, n.2, p. 155-168, 1987. https://doi.org/10.1007 BF02370385
TOMINAGA, J.; SHIMADA, H.; KAWAMITSU, Y. Direct measurement of intercellular $\mathrm{CO}_{2}$ concentration in a gasexchange system resolves overestimation using the standard method. Journal of Experimental Botany, v.69, n.8, p.1981-1991, 2018. https://doi.org/10.1093/jxb/ery044

TRIFONOV, P.; LAZAROVITCH, N.; ARYE, G. Water and Nitrogen Productivity of Potato Growth in Desert Areas under Low-Discharge Drip Irrigation. Water, v.10, n.8, p.1-16, 2018. https://doi.org/10.3390/w10080970

VALENÇA， D. C.; CARVALHO, D. F.; REINERT, F.; AZEVEDO, R. A.; PINHO, C. F.; MEDICI, L. O. Automatically controlled deficit irrigation of lettuce in "organic potponics". Scientia Agricola. v.75, n.1, p.5259, 2018. https://doi.org/10.1590/1678-992x2016-0331

WULLSCHLEGER, S. D. Biochemical Limitations to Carbon Assimilation in $\mathrm{C} 3$ Plants-A Retrospective Analysis of the A/Ci Curves from 109 Species. Journal of Experimental Botany, v.44, n. 262, p.907920.

1993.

https://doi.org/10.1093/jxb/44.5.907

YURI, J.E.; MOTA, J.H.; RESENDE, G.M.; SOUZA, R.J. Nutrição e adubação da cultura da alface. In: PRADO, R. de M.; CECÍLIO FILHO, A. B. (Ed.). Nutrição e adubação de hortaliças. Jaboticabal: Faculdade de Ciências Agrárias e Veterinárias, UNESP, 2016. Cap. 21, p. 559-577.

ZHANG, H.; CHI, D.; WANG, Q.; FANG, J.; FANG, X. Yield and quality response of cucumber to irrigation and nitrogen fertilization under subsurface drip irrigation in solar greenhouse. Agricultural Sciences in China. v.10, n.6, 921-930, 2011. https://doi.org/10.1016/S1671-

2927(11)60077-1 
Barbosa et al.

ZHANG, H.; KELIN, H.; ZHANG, L.; JI, Y.; QIN, W. crops leaching
Exploring optimal catch for reducing nitrate in vegetable greenhouse in North China. Agricultural Water Management. v. 212, n. 1, 273-282, 2019. https://doi.org/10.1016/ j.agwat.2018.09.018 